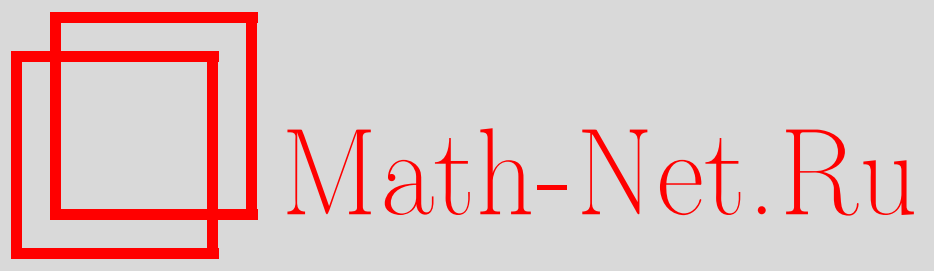

А. М. Чуднов, Алгоритмы классификации и идентификации ситуаций на основе взвешивания, Дискрет. матем., 2014, том 26, выпуск 4, 119-134

DOI: https://doi.org/10.4213/dm1310

Использование Общероссийского математического портала Math-Net.Ru подразумевает, что вы прочитали и согласны с пользовательским соглашением http://www . mathnet.ru/rus/agreement

Параметры загрузки:

IP : 52.6 .47 .48

26 апреля 2023 г., 12:52:28

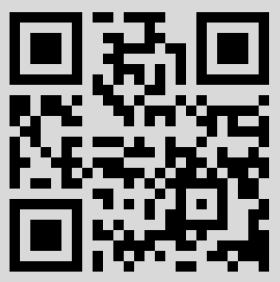




\title{
Алгоритмы классификации и идентификации ситуаций на основе взвешивания
}

\author{
() 2014 г. А. М. Чуднов*
}

\begin{abstract}
Получены нижние границы минимального числа $m$ взвешиваний, необходимых для идентификации до $t$ нестандартных объектов из общего числа $n$ тестируемых объектов. Для задачи с фиксированными отклонениями весов нестандартных объектов построены совершенные алгоритмы с параметрами $n=11, m=5, t=2$, соответствующими параметрам троичного кода Виртакаллио-Голея. Установлено несуществование совершенного кода взвешивания с такими параметрами.
\end{abstract}

Ключевые слова: взвешивание, выявление фальшивых монет, алгоритм классификации.

\section{1. Введение}

Задачи идентификации стандартных и нестандартных объектов на основе последовательных взвешиваний групп объектов обычно рассматриваются применительно к отысканию фальшивых монет [1-4].

Хорошо изучены задачи идентификации одного нестандартного объекта из общего числа $n$ объектов (в частности, с определением его относительного веса) за наименьшее число взвешиваний. В двойственной постановке соответствующие задачи состоят в определении максимального числа объектов, среди которых за данное число взвешиваний $m$ может быть идентифицирован нестандартный объект. Результаты решения этих задач подытожены в [2] в терминах фальшивых и настоящих монет, а именно (в цитируемом фрагменте изменен символ, обозначающий число взвешиваний):

"Пусть из заданного количества монет можно найти фальшивую за $m$ взвешиваний. Тогда максимальное возможное количество монет равно:

если относительный вес фальшивой монеты известен заранее $-3^{m}$ (независимо от наличия запаса настоящих монет);

если относительный вес не известен и его требуется узнать - при отсутствии запаса настоящих монет $\left(3^{m}-3\right) / 2$, при наличии $\left(3^{m}-1\right) / 2$;

если не требуется узнать относительный вес - при отсутствии запаса настоящих монет $\left(3^{m}-1\right) / 2$, при наличии $\left(3^{m}+1\right) / 2 . "$

${ }^{*}$ Место работы: Санкт-Петербургский государственный университет телекоммуникаций им. М.И. Бонч-Бруевича, e-mail: chudnow@yandex.ru 
Эти результаты соответствуют постановке задачи, в которой заранее известно, что в тестируемой группе объектов находится ровно один нестандартный объект. Впрочем, данные оценки легко модифицировать на случай, когда не известно, есть нестандартный объект среди исследуемых или нет.

Рассматриваемые задачи тесно связаны с задачами построения помехоустойчивых кодов, вообще говоря, с обратной связью $[5,6]$. Однако такие особенности задач взвешивания, как предмет поиска (в терминах теории кодирования - не кодовые блоки, а конфигурации ошибок), специфика области неопределенности, ограничения на класс функций обратной связи и другие, не позволяют напрямую использовать здесь результаты теории помехоустойчивого кодирования. Вместе с тем при $t=1$ во всех отмеченных выше случаях оптимальный алгоритм идентификации (алгоритм взвешивания) может быть построен на основе кодов без обратной связи, т. е. в так называемом статическом виде. При этом проверочные соотношения можно получить из подходящих линейных кодов над $G F(3)$ (см п.2).

В настоящей работе изучается обобщение задачи взвешивания на случай классификации и идентификации ситуаций в некотором множестве, описывающем неопределенность условий поиска. В качестве приложения рассматривается задача идентификации нестандартных объектов в следующей постановке.

Пусть в тестируемой группе, состоящей из $n$ объектов, может быть не более $t$ нестандартных объектов (остальные стандартные). Веса нестандартных объектов могут отличаться от веса стандартного на неизвестную фиксированную величину в большую или меньшую сторону. Необходимо определить наименьшее число взвешиваний $m$, необходимое и достаточное для гарантированной идентификации типов (стандартный/нестандартный) всех объектов, и построить соответствующий алгоритм взвешивания (AB).

В работе установлено, что в рассматриваемом классе задач AB, идентифицирующий типы объектов, в то же время определяет относительные отклонения весов этих объектов. Для произвольных значений $n, t$ получены нижние границы минимального числа взвешиваний $m$ для идентификации не более $t$ нестандартных объектов из группы $n$ объектов.

Построены совершенные алгоритмы с параметрами $n=11, m=5, t=2$, соответствующими параметрам троичного кода, открытого Виртакаллио и Голеем [8]. В то же время установлено несуществование совершенного кода взвешивания с такими параметрами даже при возможности использования неограниченного числа эталонных объектов.

\section{2. Основные определения}

Пусть $\mathbb{R}^{n}-n$-мерное евклидово пространство, $\left[\boldsymbol{e}^{1}, \boldsymbol{e}^{2}\right]-$ скалярное произведение векторов $\boldsymbol{e}^{1}$ и $\boldsymbol{e}^{2}$ из $\mathbb{R}^{n}, \mathbf{0}=(0,0, \ldots, 0)$ и $\mathbf{1}=(1,1, \ldots, 1)$ - выделенные элементы $\mathbb{R}^{n}$. Запись $e=e^{1} e^{2}$ означает, что вектор $e$ образован покомпонентным умножением векторов $\boldsymbol{e}^{1}$ и $\boldsymbol{e}^{2}$, записи $\left(\boldsymbol{e}^{1}, \boldsymbol{e}^{2}\right),\left(e, \boldsymbol{e}^{1}\right),\left(\boldsymbol{e}^{1}, e\right)$ используются для обозначения соответствующих конкатенаций. Мощность множества $E \subseteq \mathbb{R}^{n}$ обозначается символом $|E|$. Для элементов $e \in \mathbb{R}^{n}$ и подмножеств $E \subseteq \mathbb{R}^{n}$ используются операции $(\cdot)^{*}$ и $(\cdot)^{+}$, результаты применения которых к векторам $\boldsymbol{e}=\left(e_{1}, \ldots, e_{n}\right)$ и множествам $E=\left\{e^{j}\right\}$ определяются соотношениями $\boldsymbol{e}^{*}=\left(\operatorname{sign}\left(e_{i}\right)\right)_{i}, E^{*}=\left\{\left(\mathbf{e}^{j}\right)^{*}\right\}$, $\mathbf{e}^{+}=\left(\left|\operatorname{sign}\left(e_{i}\right)\right|\right)_{i}, E^{+}=\left\{\left(\mathbf{e}^{j}\right)^{+}\right\}$. При этом величина $\left[\mathbf{e}^{+}, \mathbf{1}\right]$ равна весу Хемминга последовательности $\mathbf{e}=\left(e_{1}, \ldots, e_{n}\right)$ и обозначается $w(\mathbf{e})=\left[\mathbf{e}^{+}, \mathbf{1}\right]$. 
Символом $I^{n}$ обозначается дискретный $[-1,1]$-куб в $\mathbb{R}^{n}$, т. е. множество всех последовательностей длины $n$ над алфавитом $I=\{-1,0,1\}$, а подмножество $I_{t}^{n} \subseteq$ $I^{n}$, определенное условием $I_{t}^{n}=\left\{\mathbf{x} \in I^{n} \mid w(\mathbf{x}) \leqslant t\right\}$, представляет собой дискретный шар радиуса $t$ (в метрике Хемминга) с центром в точке $\mathbf{0}$.

Пусть имеется $n$ объектов, относительные веса которых задаются вектором $\mathbf{x}=$ $\left(x_{1}, \ldots, x_{n}\right) \in I^{n}$, определяющим конфигурацию весов объектов: $i$-й объект имеет стандартный вес, если $x_{i}=0$; вес $i$-го объекта отличается на постоянную (неизвестную) величину в большую (меньшую) сторону, если $x_{i}=1$ (соответственно $\left.x_{i}=-1\right)$. Вектор $\mathbf{x}^{+}$характеризует типы объектов: стандартный, нестандартный (т. е. конфигурацию типов) и не содержит информации об относительных весах нестандартных объектов.

Взвешивание (проверка) задается вектором $\mathbf{h} \in I^{n}$, при этом результат взвешивания для ситуации $\mathbf{x} \in I^{n}$ равен $s(\mathbf{x}, \mathbf{h})=\operatorname{sign}([\mathbf{x}, \mathbf{h}])$. Взвешивание, определенное вектором $\mathbf{h}=\left(h_{1}, \ldots, h_{n}\right)$, имеет следующую интерпретацию: при данной проверке $i$-й объект участвует во взвешивании, если $h_{i} \neq 0$; он кладется на левую чашку весов, если $h_{i}<0$ и на правую - если $h_{i}>0$. При каждом взвешивании $\mathbf{h}$ на обеих чашках должно быть одинаковое число объектов: недостающее на какой-либо чашке число объектов пополняется эталонными объектами, число которых равно $r(\mathbf{h})=[\mathbf{h}, \mathbf{1}]$. Результат взвешивания $s(\mathbf{x}, \mathbf{h})$ описывает случаи: при $s(\mathbf{x}, \mathbf{h})=0-$ равновесие, при $s(\mathbf{x}, \mathbf{h})=-1$ - левая чашка перевешивает, при $s(\mathbf{x}, \mathbf{h})=1$ - правая чашка перевешивает. Взвешивание, не использующее дополнительных эталонных объектов $(r(\mathbf{h})=0)$, называется центрированным.

Неполнота исходной информации относительно распределения весов рассматриваемой группы объектов характеризуется множеством допустимых распределений весов объектов $Z \subseteq I^{n}$, которое также называется множеством допустимых ситуаций, элементы $\mathbf{z} \in Z$ называются допустимыми ситуациями.

Каждое взвешивание $\mathbf{h}$ определяет разбиение множества $I^{n}$ плоскостью $[\mathbf{x}, \mathbf{h}]=0$ на три части $W\left(s \mid I^{n}, \mathbf{h}\right)=\left\{\mathbf{x} \in I^{n} \mid s(\mathbf{x}, \mathbf{h})=s\right\}, s \in I$, и соответствующее разбиение множества $Z=W(0 \mid Z, \mathbf{h})+W(1 \mid Z, \mathbf{h})+W(-1 \mid Z, \mathbf{h})$, где $W(s \mid Z, \mathbf{h})=W\left(s \mid I^{n}, \mathbf{h}\right) \cap$ $Z$. C учетом этого будем говорить, что взвешивание $\mathbf{h}$ классифицирует элементы $\mathbf{z} \in Z$ по типам, соответствующим подмножествам $W(s \mid Z, \mathbf{h})$; при этом величина $d(Z, \mathbf{h})=\max _{s \in I}|W(s \mid Z, \mathbf{h})|$ называется диаметром классификации множества $Z$ взвешиванием $\mathbf{h}$. Взвешивание $\mathbf{h}=\mathbf{0}$ будем называть фиктивным. Для фиктивного взвешивания, очевидно, $s(\mathbf{z}, \mathbf{0})=0$ и $W(0 \mid Z, \mathbf{0})=Z$.

Для обозначения последовательностей, составляющих алгоритмы взвешивания (см. ниже определение 1 ), используются скобки $\langle\cdot\rangle$; далее такое отличие от обозначения последовательностей $\mathbf{x}=\left(x_{1}, \ldots, x_{n}\right)$ трактуется в смысле $\langle\mathbf{x}\rangle=(\mathbf{x})^{T}(T-$ знак транспонирования).

Определение 1. Алгоритм взвешивания $\mathcal{A}$ длины $m$ - это последовательность $\mathcal{A}=\left\langle\mathcal{A}_{1}, \ldots, \mathcal{A}_{m}\right\rangle$, где $\mathcal{A}_{j}: I^{j-1} \rightarrow I^{n}-$ функция, определяющая проверку $\mathbf{h}^{j}=\mathcal{A}_{j}\left(\mathbf{s}^{j-1}\right), \mathbf{h}^{j} \in I^{n}$, на каждом $j$-м $, j=1,2, \ldots, m$, шаге алгоритма по результатам $\mathbf{s}^{j-1}=\left(s_{1}, \ldots, s_{j-1}\right) \in I^{j-1}$ взвешиваний на предыдущих шагах $\left(\mathbf{h}^{1}=\mathcal{A}_{1}()-\right.$ заданная исходная проверка).

Работа $\mathrm{AB} \mathcal{A}$ при проверке ситуации $\mathbf{z} \in Z$ происходит следующим образом: на первом шаге по ранее определенной проверке $\mathbf{h}^{1}=A_{1}()$ вычисляется результат взвешивания $s_{1}=s\left(\mathbf{z}, \mathbf{h}^{1}\right), \mathbf{s}^{1}=\left(s_{1}\right) \in I^{1}$; на каждом последующем $j$-м шаге, 
$j=2, \ldots, m$, определяются взвешивания $\mathbf{h}^{j}=A_{j}\left(\mathbf{s}^{j-1}\right), \mathbf{h}^{j} \in I^{n}$, и вычисляется их результат $s_{j}=s\left(\mathbf{z}, \mathbf{h}^{j}\right), \mathbf{s}^{j}=\left(\mathbf{s}^{j-1} s_{j}\right)$.

Последовательность проверок $\mathcal{A}(\mathbf{z})=\left\langle\mathbf{h}^{1}, \ldots, \mathbf{h}^{m}\right\rangle, \quad$ где $\mathbf{h}^{j}=$ $\mathcal{A}_{j}\left(s\left(\mathbf{z}, \mathbf{h}^{1}\right), \ldots, s\left(\mathbf{z}, \mathbf{h}^{j-1}\right)\right), \quad$ алгоритмом $\mathcal{A}$ для ситуации $\mathbf{z}$ называется процедурой работъ $A B \mathcal{A}$ в ситуации z. Соответствующая последовательности проверок $\mathcal{A}(\mathbf{z})$ последовательность результатов взвешиваний для вектора $\mathbf{z}$ называется синдромом ситуации $\mathbf{z}$, который обозначается $\mathbf{s}(\mathbf{z} \mid \mathcal{A})=\left(s\left(\mathbf{z}, \mathbf{h}^{1}\right), \ldots, s\left(\mathbf{z}, \mathbf{h}^{m}\right)\right)$. Последовательность $\mathbf{s}=\left(s_{1}, \ldots, s_{m}\right) \in I^{m}$ называется $(Z, \mathcal{A})$-синдромом, если она является синдромом некоторой ситуации $\mathbf{z} \in Z$.

Как видно, приведенное определение АВ предполагает одинаковую длину процедур взвешивания для всех ситуаций $\mathbf{z} \in Z$. Однако в рамках данного определения могут быть описаны также алгоритмы с различными длинами процедур проверки различных ситуаций: для этого более короткие последовательности проверок могут быть пополнены фиктивными проверками.

Число используемых в процедуре $\mathcal{A}(\mathbf{z})=\left\langle\mathbf{h}^{1}, \ldots, \mathbf{h}^{m}\right\rangle$ дополнительных эталонных объектов определяется величиной $r(\mathcal{A}(\mathbf{z}))=\max \left\{r\left(\mathbf{h}^{1}\right), \ldots, r\left(\mathbf{h}^{m}\right)\right\} ;$ при этом для алгоритма $\mathcal{A}$ соответствующая величина определяется условием $r(\mathcal{A})=$ $\max _{\mathbf{z} \in Z} r(\mathcal{A}(\mathbf{z}))$.

Подалгоритмом длины $l$ алгоритма взвешивания $\mathcal{A}=\left\langle\mathcal{A}_{1}, \ldots, \mathcal{A}_{m}\right\rangle$ будем называть такой $\mathrm{AB} \mathcal{B}=\left\langle\mathcal{B}_{1}, \ldots, \mathcal{B}_{l}\right\rangle$, что для некоторого $\mathbf{s}^{i} \in I^{i}(0 \leqslant i \leqslant m-1)$ выполняются равенства $\mathcal{B}_{j}\left(\mathbf{s}^{j-1}\right)=\mathcal{A}_{i+j}\left(\mathbf{s}^{i}, \mathbf{s}^{j-1}\right)$ при всех $j=1,2, \ldots, l$. Если при этом $i=0$, то АВ $\mathcal{B}$ будет называться начальным подалгоритмом $\mathrm{AB} \mathcal{A}$.

Пусть $S(Z, \mathcal{A})$ - множество всех $(Z, \mathcal{A})$-синдромов, $W(\mathbf{s} \mid \mathcal{A}) \subseteq I^{n}$ - множество ситуаций, имеющих одинаковый синдром s, т. е. $W(\mathbf{s} \mid \mathcal{A})=\left\{\mathbf{z} \in I^{n} \mid \mathbf{s}(\mathbf{z} \mid \mathcal{A})=\mathbf{s}\right\}$; $W(\mathbf{s} \mid Z, \mathcal{A})=W(\mathbf{s} \mid \mathcal{A}) \cap Z$. Поскольку из $\mathbf{s}^{1} \neq \mathbf{s}^{2}$ следует $W\left(\mathbf{s}^{1} \mid \mathcal{A}\right) \cap W\left(\mathbf{s}^{2} \mid \mathcal{A}\right)=\oslash$, то множество $S(Z, \mathcal{A})$ индуцирует разбиение множества ситуаций $Z$ на подмножества $W(\mathbf{s} \mid \mathcal{A}) \subseteq Z, \mathbf{s} \in S(Z, \mathcal{A})$. Таким образом, алгоритм $\mathcal{A}$ классифицирует ситуации из $Z$ по подмножествам $W(\mathbf{s} \mid \mathcal{A}), \mathbf{s} \in S(Z, \mathcal{A})$.

Спектром классификации ситуащий (типов обгектов) алгоритма $\mathcal{A}$ в $Z$ называется заданная на множестве $S(Z, \mathcal{A})$ функция $M(\mathbf{s})=M(\mathbf{s} \mid Z, \mathcal{A})=|W(\mathbf{s} \mid Z, \mathcal{A})|$ $\left(\right.$ соответственно $\left.M^{+}(\mathbf{s})=M^{+}(\mathbf{s} \mid Z, \mathcal{A})=\left|W^{+}(\mathbf{s} \mid Z, \mathcal{A})\right|\right)$. С учетом этого диаметры классификации ситуаций и типов объектов определяются соответственно соотношениями: $\left.d(Z, \mathcal{A})=\max _{\mathbf{s} \in S(Z, \mathcal{A})} M(\mathbf{s} \mid Z, \mathcal{A}), d^{+}(Z, \mathcal{A})=\max _{\mathbf{s} \in S(Z, \mathcal{A})} M^{+}(\mathbf{s} \mid Z, \mathcal{A})\right)$.

\section{Определение 2. АВ $\mathcal{A}$ называется}

а) идентифицирующим ситуации в множестве $Z$, если для всех $\mathbf{s} \in S(Z, \mathcal{A})$ выполняется условие $|W(\mathbf{s} \mid Z, \mathcal{A})|=1$;

b) идентифицирующим типь обгектов в множестве $Z$, если для всех $\mathbf{s} \in$ $S(Z, \mathcal{A})$ выполняется условие $\left|W^{+}(\mathbf{s} \mid Z, \mathcal{A})\right|=1$.

В соответствии с определением алгоритм, идентифицирующий ситуации (типы объектов), по синдрому $\mathbf{s}$ определяет единственную ситуацию $\mathbf{z} \in W(\mathbf{s} \mid Z, \mathcal{A})$ (соответственно, единственную конфигурацию типов $\left.\mathbf{z}^{+} \in W^{+}(\mathbf{s} \mid Z, \mathcal{A})\right)$. Будем также говорить, что АВ, идентифицирующий ситуации, идентифицирует относительные веса тестируемых объектов.

Определение 3. $\mathrm{AB} \mathcal{A}=\left\langle\mathcal{A}_{1}, \ldots, \mathcal{A}_{m}\right\rangle$ называется статическим, или кодом взвешивания, если при всех $j=1,2, \ldots, m$ проверки $\mathbf{h}^{j}=\mathcal{A}_{j}\left(\mathbf{s}^{j-1}\right)=\mathcal{A}_{j}()$ не зависят от значений $\mathbf{s}^{j-1}$, в противном случае $\mathrm{AB} \mathcal{A}$ называется динамическим. 
Код взвешивания (KB) представляет собой заранее определенную последовательность проверок $\left\langle\mathbf{h}^{1}, \ldots, \mathbf{h}^{m}\right\rangle$. Поскольку при описании свойств КВ используются матричные операции, постольку код отождествляется с $(m, n)$-матрицей $H=\left(h_{i}^{j}\right)_{j i}$, строки которой $\mathbf{h}^{j}=\left(h_{1}^{j}, \ldots, h_{n}^{j}\right), j=1,2, \ldots, m$, определяют взвешивания, а последовательности $\mathbf{h}_{i}=\left(h_{i}^{1}, \ldots, h_{i}^{m}\right), i=1,2, \ldots, n$, соответствующие ее столбцам $\mathbf{h}_{i}^{T}$, определяют элементы, участвующие в проверках. Матрицу $\mathbf{H}$ будем называть проверочной матрицей кода или просто матрицей кода.

Алгоритмы и коды взвешивания длины $m$, идентифицирующие ситуации в множестве $Z=I_{t}^{n}$ и использующие во взвешиваниях не более $r$ эталонных объектов, будем называть соответственно $(n, m, t, r)$-алгоритмами (кодами), а при $r=0-$ $(n, m, t)$-алгоритмами (кодами).

В качестве примеров при $t=1$ укажем коды $\mathbf{K}_{1}, \mathbf{K}_{2}, \mathbf{K}_{3}$, где $(n, m, 1,1)$-код $\mathbf{K}_{1}$ описывается матрицей проверок, содержащей в качестве столбцов набор $n=$ $\left(3^{m}-1\right) / 2$ попарно линейно независимых векторов из $I^{m}$. Проверочная матрица KB $\mathbf{K}_{1}$ соответствует проверочной матрице кода Хемминга над $G F(3)$ с алфавитом $I$ и теми же параметрами. При удалении определенного столбца в указанной матрице код $\mathbf{K}_{1}$ легко превращается в $(n-1, m, 1)$-код $\mathbf{K}_{2}$. Коды $\mathbf{K}_{1}, \mathbf{K}_{2}$ идентифицируют относительные веса объектов в множестве ситуаций $I_{1}^{n}$. Если множество столбцов проверочной матрицы кода $\mathbf{K}_{2}$ пополнить $\mathbf{0}$-вектором, то полученный в результате код $\mathbf{K}_{3}$ будет идентифицировать типы объектов в множестве $Z=I_{1}^{n} \backslash\{\mathbf{0}\}$. Приведенные примеры представляют решения описанных во введении задач с одним нестандартным объектом.

\section{3. Необходимые условия существования и нижние границы минимального числа взвешиваний}

Утверждения, на которые в дальнейшей части работы делаются ссылки, формулируются в виде отдельных предложений. Очевидные, а также вытекающие из предыдущих заключений предложения приводятся без доказательств.

В общем случае необходимые условия существования АВ могут быть получены на основе следующих очевидных неравенств.

Предложение 1. а) $\left.d(Z, \mathcal{A}) \geqslant|Z| / 3^{m} ; b\right) d^{+}(Z, \mathcal{A}) \geqslant\left|Z^{+}\right| / 3^{m}$.

Из данных утверждений и определения 2 немедленно вытекает следующее.

Предложение 2. Длина $A B$, идентифицирующего а) ситуации, $b$ ) типы объектов в множестве $Z$, удовлетворяет соответственно неравенствам a) $m \geqslant \log _{3}(|Z|)$, b) $m \geqslant \log _{3}\left(\left|Z^{+}\right|\right)$.

Ниже устанавливается, что в весьма широком классе задач АВ, идентифицирующие типы объектов, идентифицируют также относительные веса объектов. Введем дополнительные понятия и сформулируем предварительные утверждения, на основе которых далее будет получен этот результат.

Определение 4. Подобными называются векторы $\mathbf{x}, \mathbf{y} \in I^{n}$, для которых $\mathbf{x}^{+}=\mathbf{y}^{+}$. 
В соответствии с определением для подобных векторов $\mathbf{x}=\left(x_{1}, \ldots, x_{n}\right), \mathbf{y}=$ $\left(y_{1}, \ldots, y_{n}\right)$ справедливо: $x_{i} \neq y_{i} \Rightarrow x_{i} y_{i}=-1$ и, следовательно, $(\mathbf{x}+\mathbf{y}) / 2 \in I^{n}$. Любое из этих условий может быть положено в основу определения подобных векторов.

Определение 5. Множество $X \subseteq I^{n}$ называется подходящим, если для любых подобных векторов $\mathbf{x}, \mathbf{y} \in X$ выполняется условие $(\mathbf{x}+\mathbf{y}) / 2 \in X$.

Несколько простых примеров:

1. Пустое множество и все одноэлементные множества $\{\mathbf{x}\}$ являются подходящими.

2. Множество $\{(0,1),(1,0),(1,1),(-1,1)\} \subseteq I^{2}$ является подходящим.

3. $I^{n}$ и $I_{t}^{n}-$ подходящие множества.

4. Множество $I_{t}^{n} \backslash\{\boldsymbol{0}\}$ при $n \geqslant 1$ и $t \geqslant 1$ не является подходящим.

Пустое и одноэлементные подходящие множества будем называть тривиальнъми.

Предложение 3. Если $X$ - нетривиальное подходящее множество, то $\left|X^{+}\right| \geqslant 2$.

Доказательство. Пусть $\mathbf{x}, \mathbf{y} \in X-$ подобные векторы $(\mathbf{x} \neq \mathbf{y})$ и для вектора $\mathbf{z} \in X$ выполняется равенство $\mathbf{z}=(\mathbf{x}+\mathbf{y}) / 2$. Тогда $x_{i}=-y_{i}$ при некотором $i$ и, следовательно, $z_{i}=0$. Таким образом, $\mathbf{z}^{+} \neq \mathbf{x}^{+}$и $\mathbf{z}^{+} \neq \mathbf{y}^{+}$, откуда следует, что $\left|X^{+}\right| \geqslant 2$.

Предложение 4. Пересечение подходящих множеств есть подходящее множество.

Утверждение непосредственно следует из определения 6.

Как видно, введенное понятие подходящего множества может быть вписано в обобщенное понимание дискретного выпуклого множества $[8,9]$.

Теорема 1. Пусть $Z \subseteq I^{n}-$ подходящее множество. Тогда $A B \mathcal{A}$, идентифицирующий типь обгектов в множестве $Z$, идентифицирует ситуации в $Z$.

Доказательство. Для $(Z, \mathcal{A})$-синдрома $\mathbf{s}=\left(s_{1}, \ldots, s_{m}\right) \in S(Z, \mathcal{A})$ представим множество $W(\mathbf{s} \mid Z, \mathcal{A})$ в виде $W(\mathbf{s} \mid Z, \mathcal{A})=Z \cap W\left(s_{1}\right) \cap W\left(s_{2}\right) \ldots \cap W\left(s_{m}\right)$, где $W\left(s_{j}\right)=\left\{\mathbf{x} \in I^{n} \mid s\left(\mathbf{x}, \mathbf{h}^{j}\right)=s_{j}\right\}$. Из последнего соотношения с учетом предложения 4 и условия теоремы следует, что $W(\mathbf{s} \mid Z, \mathcal{A})$ - подходящее множество. Поскольку $\left|W^{+}(\mathbf{s} \mid Z, \mathcal{A})\right|=1$ (см. определение 2$)$, то $|W(\mathbf{s} \mid Z, \mathcal{A})|=1$ в силу предложения 3 , т. е. $\mathrm{AB} \mathcal{A}$ идентифицирует ситуации, что требовалось доказать.

Далее полагается, что $Z \subseteq I^{n}-$ подходящее множество. Так как в этом случае все типы классификации (идентификации) совпадают, то будем говорить об АВ, классифицирующих (идентифицирующих) ситуации.

Следующее утверждение является непосредственным следствием предложения 2, и с учетом теоремы 1 оно распространяется на алгоритмы, идентифицирующие типы объектов.

Предложение 5. Если существует $(n, m, t, r)-A B$, mo $m \geqslant \log _{3}\left(\sum_{i=0}^{t} 2^{i} C_{n}^{i}\right)$.

Как видно, условие предложения 5 соответствует границе Хемминга для числа проверочных элементов в коде, исправляющем ошибки кратности не больше $t$ над троичным алфавитом. 
Определение 6. Совершенным алгоритмом классификации (в частности, идентификации) в $Z$ называется $\mathrm{AB} \mathcal{A}$, для которого $d(Z, \mathcal{A})=|Z| / 3^{m}$.

Для совершенного алгоритма идентификации в $Z$, очевидно, $d(Z, \mathcal{A})=1$ и $|Z|=3^{m}$.

Каждый начальный подалгоритм совершенного $\mathrm{AB}$ в $Z$ является совершенным алгоритмом в $Z$. Любой подалгоритм, входящий в состав совершенного АВ на $j$-м шаге, является совершенным в одном или нескольких множествах $W(s \mid Z, \mathcal{A}), s \in$ $S\left(Z, \mathcal{A}^{j-1}\right)$. В частности, взвешивание можно рассматривать как алгоритм (код) классификации длины 1 , при этом каждое взвешивание в совершенном алгоритме классифицирует множество ситуаций на 3 равномощные подмножества. Любой подкод совершенного кода взвешивания в $Z$ является совершенным в $Z$.

\section{4. Некоторые свойства проверочных матриц кодов взвешивания в $I_{t}^{n}$}

AВ могут строиться на основе включения в их состав в качестве подалгоритмов нетривиальных кодов взвешивания. С другой стороны, КВ могут выступать в качестве самостоятельных алгоритмов классификации (идентификации) ситуаций.

Для описания кодов взвешивания будем использовать понятия эквивалентных алгоритмов и допустимых преобразований, задаваемых операциями умножения на матрицы. На основе введенных здесь понятий и рассмотренных свойств кодов в п. 5 строятся и описываются совершенные $(11,5,2)$-алгоритмы взвешивания.

Пусть $\mathcal{G}_{p}^{n}$ - множество (группа) перестановочных $(n, n)$-матриц, $\mathcal{G}_{d}^{n}-$ множество (группа) диагональных $(n, n)$-матриц с диагональными элементами $c_{i i} \in\{-1,1\}$, $\mathcal{G}^{n}=\mathcal{G}_{p}^{n} \times \mathcal{G}_{d}^{n}$. Элементы из $\mathcal{G}^{n}$ и $\mathcal{G}^{m}$ рассматриваются как операторы над векторами и матрицами. Кроме того, действие операторов $\mathbf{G} \in \mathcal{G}^{n}$ распространяется также на множества векторов (последовательностей) $X=\left\{\mathbf{x}^{j}\right\}$ следующим образом: $X \mathbf{G}=$ $\left\{\mathbf{x}^{j} \mathbf{G}\right\}$.

Определение 7. а) Множества $X \subseteq I^{n}$ и $Y \subseteq I^{n}$ называются эквивалентными, если $X \mathbf{G}=Y$ для некоторого преобразования $\mathbf{G} \in \mathcal{G}^{n}$.

b) Классы множеств $\left\{X^{j}\right\}$ и $\left\{Y^{j}\right\}$ называются эквивалентными, если существует такая биекция $\left\{X^{j}\right\} \leftrightarrow\left\{Y^{j}\right\}$, что множества каждой пары $X^{j} \leftrightarrow Y^{j}$ эквивалентны.

с) Алгоритмы $\mathcal{A}, \mathcal{B}$, определенные на эквивалентных множествах $X$ и $Y$ соответственно, называются функиионально эквивалентными, если разбиения $\{W(\mathbf{s} \mid X, \mathcal{A})\}_{\mathbf{s} \in S(X, \mathcal{A})}$ и $\{W(\mathbf{s} \mid Y, \mathcal{B})\}_{\mathbf{s} \in S(Y, \mathcal{B})}$ эквивалентны.

Преобразования АВ, результат применения которых дает $\mathrm{AB}$, функционально эквивалентные исходному, называются допустимыми.

Следует заметить, что введенное понятие функциональной эквивалентности основывается лишь на результате преобразования исходного множества допустимых ситуаций $Z$ в множества $\{W(\mathbf{s} \mid Z, \mathcal{A})\}_{\mathbf{s} \in S(Z, \mathcal{A})}$ и не учитывает свойства процедуры взвешиваний. При этом, в частности, не принимается во внимание число используемых во взвешиваниях дополнительных эталонных объектов. Так, все АВ одинаковой длины, идентифицирующие ситуации в эквивалентных множествах, по определению являются функционально эквивалентными. 
Будем рассматривать совокупность преобразований кода, порожденных операторами умножения матрицы $\mathbf{H}=\left(h_{i}^{j}\right)_{j i}$ кода на матрицы $\mathbf{L} \in \mathcal{G}^{m}, \mathbf{R} \in \mathcal{G}^{n}$, соответственно слева и справа. При этом: а) операторы $\mathbf{L} \in \mathcal{G}_{p}^{m}$ осуществляют перестановку строк матрицы кода, b) операторы $\mathbf{L} \in \mathcal{G}_{d}^{m}$ умножают строки матрицы кода на $c_{i i} \in\{-1,1\}$, c) $\mathbf{R} \in \mathcal{G}_{p}^{n}$ осуществляют перестановку столбцов матрицы кода d) операторы $\mathbf{R} \in \mathcal{G}_{d}^{n}$ умножают столбцы матрицы кода на $c_{i i} \in\{-1,1\}$. В частности, умножение всех элементов матрицы $\mathbf{H}$ на -1 соответствует умножению $\mathbf{H}$ как слева на $-\mathbf{1} \in \mathcal{G}_{d}^{m}$, так и справа на $-\mathbf{1} \in \mathcal{G}_{d}^{n}$ и обозначается $(-1) \mathbf{H}=\mathbf{H}(-1)=-\mathbf{H}$. Действие операторов $\mathbf{L} \in \mathcal{G}^{m}, \mathbf{R} \in \mathcal{G}^{n}$ на множество векторов (последовательностей) $X=\left\{\mathbf{x}^{i}\right\}, \mathbf{x}^{i} \in I^{i}$, понимается в смысле $\mathbf{L} X=\left\{\mathbf{L} \mathbf{x}^{i}\right\}, X \mathbf{R}=\left\{\mathbf{x}^{i} \mathbf{R}\right\}$. Далее полагается $Z=I_{t}^{n}$, при этом, учитывая, что множество $Z$ инвариантно относительно преобразований $\mathbf{G} \in \mathcal{G}^{n}$, в записи $W(\mathbf{s} \mathbf{L} \mid Z, \mathbf{H})$ аргумент $Z$ будем опускать.

Предложение 6. Если $\boldsymbol{L} \in \mathcal{G}^{m}, m o W(s \boldsymbol{L} \mid \boldsymbol{H})=W(s \mid \boldsymbol{L} \boldsymbol{H})$.

При $\mathbf{L}=-\mathbf{1}$ отсюда получим $W(-\mathbf{s} \mid \mathbf{H})=W(\mathbf{s} \mid-\mathbf{H})$.

Предложение 7. Если $\boldsymbol{R} \in \mathcal{G}^{n}, \operatorname{mo} W(s \mid \boldsymbol{H}) \boldsymbol{R}=W(s \mid \boldsymbol{H R})$.

При $\mathbf{R}=-\mathbf{1}$ отсюда получим $-W(\mathbf{s} \mid \mathbf{H})=W(\mathbf{s} \mid-\mathbf{H})$, и с учетом предыдущего предложения $W(-\mathbf{s} \mid \mathbf{H})=-W(\mathbf{s} \mid \mathbf{H})$.

При $\mathbf{L} \in \mathcal{G}^{m}, \mathbf{R} \in \mathcal{G}^{n}$ коды $\mathbf{H}^{1}$ и $\mathbf{H}^{2}=\mathbf{L} \mathbf{H}^{1} \mathbf{R}$ функционально эквивалентны, т. е. все допустимые преобразования сохраняют функциональную эквивалентность кода. При $\mathbf{L} \in \mathcal{G}^{m}, \mathbf{R} \in \mathcal{G}_{p}^{n}$ коды $\mathbf{H}^{1}$ и $\mathbf{H}^{2}$ отличаются лишь нумерацией объектов, очередностью взвешиваний и нумерацией чашек. Учитывая, что преобразования $\mathbf{L} \in \mathcal{G}^{m}, \mathbf{R} \in \mathcal{G}_{p}^{n}$ по существу не меняют алгоритм взвешивания, их будем называть условными.

Для $\mathbf{s} \in I^{m}$ обозначим через $N_{m}(\mathbf{s})$ число столбцов в матрице кода $\mathbf{H}$, равных $\mathbf{s}^{T}$ или $-\mathbf{s}^{T}$. При этом значения $N_{m}(\mathbf{s})$ определяют матрицу кода с точностью до допустимых преобразований.

Вектор $\mathbf{s} \in I^{m}$, у которого ненулевой элемент с наименьшим номером координаты равен 1, будем называть приведенным. Приведенной называется матрица, столбцы которой являются приведенными векторами.

Предложение 8. Матрица КВ может быть преобразована к приведенному виду допустимыми преобразованиями.

С учетом данного утверждения для построения $\mathrm{KB}$ при $r=n$ и установления факта существования/несуществования кода достаточно рассматривать лишь коды с приведенными матрицами.

Для любой подматрицы кода, содержащей $k$ строк, на основе непосредственного подсчета параметров спектра классификации $M_{m}(\mathbf{s})=|W(\mathbf{s} \mid \mathbf{H})|, s \in I^{m}$, можно получить общую систему нелинейных уравнений, определяющих эти параметры через параметры $N_{m}(\mathbf{s}), \mathbf{s} \in I^{m}$, матрицы кода.

Пусть $Q_{m}(i, \mathbf{s}) \subseteq I^{m}$ обозначает множество наборов $\mathbf{q}^{i}=\left(\mathbf{u}^{1}, \ldots, \mathbf{u}^{i}\right)$ векторов $\mathbf{u}^{j} \in I^{m}, j=1,2, \ldots, i$, для которых при некотором наборе коэффициентов $c_{j} \in$ $\{-1,1\}$ выполняется условие $\left(\sum_{j=1}^{i} c_{j} \mathbf{u}^{j}\right)^{*}=\mathbf{s}$. Тогда

$$
M_{m}(\mathbf{s})=\sum_{i=1}^{t} \sum_{\mathbf{q}^{i} \in Q_{m}(i, \mathbf{s})} N_{m}\left(\mathbf{u}^{1}\right) N_{m}\left(\mathbf{u}^{2}\right) \cdots N_{m}\left(\mathbf{u}^{i}\right) .
$$

Из рассмотренного вытекает следующее утверждение. 
Предложение 9. Для существования кода взвешивания со спектром классификаици $M_{m}(s), s \in I^{m}$, необходимо и достаточно, чтобы система уравнений (1) имела решения в цельх неотрицательных числах $N_{m}\left(\boldsymbol{u}^{j}\right), j=1,2, \ldots, i$, где $\boldsymbol{u}^{j} \in I^{m}-$ приведенные векторы.

Соотношения (1), очевидно, справедливы также для любой подматрицы матрицы $\mathbf{H}$, образованной ее произвольными $k$ строками $(k=1, \ldots, m)$. Это обстоятельство позволяет решение системы уравнений (1) осуществлять рекуррентно по числу используемых в (1) строк исходной матрицы с учетом линейной зависимости параметров $N_{m}\left(\mathbf{s}^{i}\right)$ подматриц кода различных размерностей.

С целью формального описания указанной рекуррентной процедуры введем обозначения: $I^{m}(\omega)$ - множество последовательностей длины $m$ над алфавитом $I(\omega)=$ $I \cup\{\omega\}$, где $\omega$ - дополнительный символ, имеющий смысл "любой элемент из $I$ ", для $s \in I^{m}(\omega)$ положим

$$
U(s)=\left\{\mathbf{u}=\left(u_{1}, \ldots, u_{m}\right) \in I^{m} \mid \forall j \in\{1, \ldots, m\}: u_{i}=s_{i} \vee s_{i}=\omega\right\} .
$$

Тогда в общем случае справедливы равенства

$$
N_{m}(s)=\sum_{u \in U(s)}^{i} N_{m}(u), \quad M_{m}(s)=\sum_{u \in U(s)}^{i} M_{m}(u) .
$$

Принцип реализации рекуррентной вычислительной процедуры на основе системы уравнений (2) иллюстрируется в следующем параграфе на примере построения совершенных кодов классификации, на основе которых строится совершенный (11,5,2)-алгоритм идентификации.

\section{5. Построение совершенного $(11,5,2)$-алгоритма}

В силу выполнения равенства $\left|I_{2}^{11}\right|=3^{5}$, соответствующего необходимому условию существования совершенного алгоритма идентификации с параметрами $n=11, m=$ $5, t=2$, возникает вопрос о существовании такого алгоритма и, в частности, совершенного кода. Прежде всего рассмотрим вопрос о существовании совершенного кода классификации в $Z=I_{2}^{11}$ с максимальным числом проверок, который можно использовать в качестве начального подалгоритма $(11,5,2,11)$-АВ. При $t=2$ для $k$ строк матрицы кода уравнения (1) принимают вид

$$
\begin{aligned}
& M_{k}(\mathbf{0})=1+2 N_{k}^{2}(\mathbf{0})+\sum_{\mathbf{u} \neq \mathbf{0}} N_{k}(\mathbf{u})\left(N_{k}(\mathbf{u})-1\right), \\
& M_{k}(\mathbf{s})=\frac{1}{2} N_{k}(\mathbf{s})\left(1+N_{k}(\mathbf{s})\right)+\sum_{\left(\mathbf{u}^{1}, \mathbf{u}^{2}\right) \in Q_{m}(2, s), \mathbf{u}^{1} \neq \mathbf{u}^{2}} N_{k}\left(\mathbf{u}^{1}\right) N_{k}\left(\mathbf{u}^{2}\right) .
\end{aligned}
$$

При этом для одной (например, первой) строки такого кода $M_{1}(\mathbf{s})=\left|I_{2}^{11}\right| / 3=3^{4}$, а уравнения (2), (3) принимают вид

$$
\begin{aligned}
& N_{1}(\omega)=N_{1}(0)+N_{1}(1), \\
& M_{1}(0)=1+2 N_{1}^{2}(0)+N_{1}^{2}(1)-N_{1}(1),
\end{aligned}
$$

где $N_{1}(\omega)=n$ (уравнение для $M_{1}(1)$ исключено в силу его линейной зависимости от уравнения для $\left.M_{1}(0)\right)$. 
Легко установить, что при $n=11, M_{1}(0)=3^{4}$ множество $\mathcal{N}_{1}$ решений системы уравнений (4) содержит два решения $\mathcal{N}_{1}=\left\{N_{1}^{(1)}(1), N_{1}^{(2)}(1)\right\}=\{6,9\},\left(N_{1}(0)=\right.$ $\left.n-N_{1}(1)\right)$. Отсюда вытекает справедливость следующего утверждения.

Предложение 10. Для того, чтобы проверка $\mathbf{h} \in I^{11}$ была совершенной в $I_{2}^{11}$, необходимо и достаточно, чтобъ $w(\mathbf{h})=6$ или $w(\mathbf{h})=9$.

Другими словами, совершенная проверка может охватывать 6 или 9 объектов. В центрированном взвешивании может участвовать лишь 6 объектов - по три на каждой чашке весов.

Для $k=2$ из соотношений $(2),(3)$ для приведенной матрицы получим соответственно системы линейных и нелинейных уравнений:

$$
\begin{aligned}
& N_{2}(0,0)+N_{2}(0,1)=N_{2}(0, \omega), N_{2}(0,0)+N_{2}(1,0)=N_{2}(\omega, 0), \\
& N_{2}(1,0)+N_{2}(1,1)+N_{2}(1,-1)=N_{2}(1, \omega), N_{2}(0,1)+N_{2}(1,1)=N_{2}(\omega, 1) \text {; } \\
& M_{2}(0,0)=1+2 N_{2}^{2}(0,0)+N_{2}^{2}(0,1)+N_{2}^{2}(1,0)+N_{2}^{2}(1,1)+N_{2}^{2}(1,-1)- \\
& -N_{2}(0,1)-N_{2}(1,0)-N_{2}(1,1)-N_{2}(1,-1) \text {, } \\
& M_{2}(0,1)=N_{2}(0,1)+N_{2}(0,0) N_{2}(0,1)+\frac{1}{2} N_{2}(0,1)\left[N_{2}(0,1)-1\right]+ \\
& +N_{2}(1,1) N_{2}(1,-1)+N_{2}(1,0) N_{2}(1,1)+N_{2}(1,0) N_{2}(1,-1) \\
& M_{2}(1,0)=N_{2}(1,0)+N_{2}(0,0) N_{2}(1,0)+\frac{1}{2} N_{2}(1,0)\left[N_{2}(1,0)-1\right]+ \\
& +N_{2}(1,1) N_{2}(1,-1)+N_{2}(0,1) N_{2}(1,1)+N_{2}(0,1) N_{2}(1,-1) ; \\
& M_{2}(1,1)=N_{2}(1,1)+N_{2}(0,0) N_{2}(1,1)+\frac{1}{2} N_{2}(1,1)\left[N_{2}(1,1)-1\right]+ \\
& +N_{2}(0,1) N_{2}(1,0)+N_{2}(0,1) N_{2}(1,1)+N_{2}(1,0) N_{2}(1,1), \\
& M_{2}(1,-1)=N_{2}(1,-1)+N_{2}(0,0) N_{2}(1,-1)+\frac{1}{2} N_{2}(1,-1)\left[N_{2}(1,-1)-1\right]+ \\
& +N_{2}(0,1) N_{2}(1,0)+N_{2}(0,1) N_{2}(1,-1)+N_{2}(1,0) N_{2}(1,1),
\end{aligned}
$$

линейно зависимые уравнения из системы (6) исключены.

При этом участвующие в уравнениях (5), (6) величины $N_{2}(0, \omega), N_{2}(\omega, 0), N_{2}(1, \omega), N_{2}(\omega, 1)$ определены на предыдущем шаге итерации (при $k=1$ ) из решения системы уравнений (4), т. е. $N_{2}(1, \omega) \in \mathcal{N}_{1}, N_{2}(\omega, 1) \in \mathcal{N}_{1}$.

Учитывая, что для всех $\mathbf{s} \in I^{2}$ выполняется равенство $M_{2}(\mathbf{s})=3^{3}$, из последних двух уравнений в (6) нетрудно получить $N_{2}(1,-1)=N_{2}(1,1)$. Отсюда вытекает следующее свойство проверок совершенного кода классификации $(\mathrm{CKK})$ в $I_{2}^{11}$.

Предложение 11. Все проверки СКК в $I_{2}^{11}$ попарно ортогоналъны.

Последнее условие совместно с линейными уравнениями (6) позволяет выразить все неизвестные величины $N_{2}(0,0), N_{2}(0,1), N_{2}(1,0), N_{2}(1,1), N_{2}(1,-1)$ через величину $N_{2}(1,1)$ :

$$
\begin{aligned}
N_{2}(0,0)= & n-2 N_{2}(1,1)-N_{2}(1, \omega)-N_{2}(\omega, 1), N_{2}(0,1)=N_{2}(\omega, 1)-2 N_{2}(1,1), \\
& N_{2}(1,0)=N_{2}(1, \omega)-2 N_{2}(1,1), N_{2}(1,-1)=N_{2}(1,1) .
\end{aligned}
$$


При этом все (с точностью до допустимых преобразований) решения уравнений (5),(6), представленные в виде допустимых наборов троек чисел $\left(N_{2}(1, \omega), N_{2}(\omega, 1), N_{2}(0,0)\right)$, определены следующим множеством: $\quad \mathcal{N}_{2}=$ $\{(6,6,1),(6,6,3),(6,9,0),(6,9,2),(9,9,1)\}$.

Продолжая данную процедуру, при $k=3$ можно получить 25 наборов значений параметров $N_{3}(1, \omega, \omega), N_{3}(\omega, 1, \omega), N_{3}(\omega, \omega, 1), N_{3}(0,0, \omega), N_{3}(0, \omega, 0), N_{3}(\omega, 0,0)$, для которых существуют решения уравнений $(1),(2)$, и соответствующие этим наборам совершенные коды классификации длины 3. Представители всех классов функционально эквивалентных CKK в $I_{2}^{11}$ длины 3 , а также значения вышеуказанной последовательности параметров приведены в таблице 1.

Таблица 1

\begin{tabular}{|c|c|c|}
\hline 1) $6,6,6,3,3,3$ & 2) $6,6,6,1,1,3$ & 3) $6,6,9,1,0,0$ \\
\hline $\begin{array}{llllllllllll}0 & 0 & 0 & 0 & 0 & 1 & 1 & 1 & 1 & 1 & 1\end{array}$ & $\begin{array}{llllllllllll}0 & 0 & 0 & 0 & 0 & 1 & 1 & 1 & 1 & 1 & 1\end{array}$ & $\begin{array}{llllllllllll}0 & 0 & 0 & 0 & 0 & 1 & 1 & 1 & 1 & 1 & 1\end{array}$ \\
\hline $\begin{array}{llllllllll}0 & 0 & 0 & 1 & 1 & 0 & 0 & 1 & 1-1 & -1\end{array}$ & $\begin{array}{llllllllll}0 & 1 & 1 & 1 & 1 & 0 & 0 & 0 & 0 & 1-1\end{array}$ & $\begin{array}{llllllllll}0 & 1 & 1 & 1 & 1 & 0 & 0 & 0 & 0 & 1-1\end{array}$ \\
\hline $\begin{array}{lllllllllll}0 & 1 & 1 & 0 & 0 & 0 & 0 & 1 & -1 & 1 & -1\end{array}$ & $\begin{array}{llllllll}0 & 1 & 1-1-1 & 0 & 0 & 1-1 & 0 & 0\end{array}$ & $\begin{array}{llllllllll} & 1 & 1-1-1 & 1 & 1-1-1 & 0 & 0\end{array}$ \\
\hline 4) $6,6,9,1,2,0$ & 5) $6,6,9,3,0,2$ & 6) $6,6,9,3,2,2$ \\
\hline $0 \begin{array}{lllllllllll}0 & 0 & 0 & 0 & 0 & 1 & 1 & 1 & 1 & 1 & 1\end{array}$ & 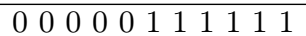 & $\begin{array}{lllllllllll} & 0 & 0 & 0 & 0 & 1 & 1 & 1 & 1 & 1 & 1\end{array}$ \\
\hline $\begin{array}{llllllllllll}0 & 1 & 1 & 1 & 1 & 0 & 0 & 0 & 0 & 1-1\end{array}$ & $\begin{array}{lllllllll}0 & 0 & 0 & 1 & 1 & 0 & 0 & 1 & 1-1-1\end{array}$ & $\begin{array}{lllllllll}0 & 0 & 0 & 1 & 1 & 0 & 0 & 1 & 1-1-1\end{array}$ \\
\hline $\begin{array}{llllllll}1 & 0 & 0 & 1-1 & 1-1-1-1 & 1 & 1\end{array}$ & $\begin{array}{lllllllll}1 & 1 & 1 & 1-1 & 0 & 0 & 1-1 & 1-1\end{array}$ & $\begin{array}{lllllllll}0 & 0 & 1 & 1-1 & 1-1 & 1-1 & 1-1\end{array}$ \\
\hline 7) $9,9,9,1,1,1$ & ---- & ---- \\
\hline $\begin{array}{llllllllllll}0 & 0 & 1 & 1 & 1 & 1 & 1 & 1 & 1 & 1 & 1 \\
0 & 1 & 0 & 1 & 1 & 1 & 1 & -1 & -1 & -1 & -1 \\
0 & 1 & -1 & 1 & 1 & -1 & -1 & 0 & 1 & 1-1\end{array}$ & ----- & ---- \\
\hline
\end{tabular}

Теорема 2. Любой $C K K$ в $I_{2}^{11}$ длины 3 функционально эквивалентен одному из семи кодов, представленных матрицами в таблице 1.

Множество всех решений системы уравнений (5), (6) и представители классов функциональной эквивалентности кодов найдены с использованием вычислительных средств.

Теорема 3. Не существует $C K K$ в $I_{2}^{11}$ длинъ 4.

Этот факт установлен путем перебора вариантов продолжения матриц кодов, представленных в таблице 1, и вычисления для получаемых кодов спектров классификации.

На основе каждого из представленных в таблице 1 кодов классификации можно построить совершенный алгоритм идентификации ситуаций в $I_{2}^{11}$, используя соответствующий код в качестве начального подалгоритма АВ. Наибольший интерес представляют $(11,5,2)$-алгоритмы идентификации (т. е. не использующие эталонных объектов), которые ниже строятся на базе представленных в таблице кодов 1) и 2). При этом в алгоритме $\mathcal{A}=\left\langle\mathcal{A}_{1}, \ldots, \mathcal{A}_{m}\right\rangle$ проверки $\mathbf{h}^{1}=\mathcal{A}_{1}, \mathbf{h}^{2}=\mathcal{A}_{2}, \mathbf{h}^{3}=\mathcal{A}_{3}$ составляют CKK $\mathbf{H}_{1}=\left\langle\mathbf{h}^{1}, \mathbf{h}^{2}, \mathbf{h}^{3}\right\rangle$ в $Z=I_{2}^{11}$, а проверки $\mathbf{h}^{4}, \mathbf{h}^{5}$ определяются по значениям синдрома $\mathbf{s}=\mathbf{s}\left(\mathbf{z} \mid \mathbf{H}_{1}\right)=\left(\mathbf{z H}_{1}^{T}\right), \mathbf{s} \in I^{3}$, и образуют коды $\mathbf{H}_{2}(\mathbf{s})=\left\langle\mathbf{h}^{4}, \mathbf{h}^{5}\right\rangle$, идентифицирующие ситуации в множествах $W\left(\mathbf{s} \mid \mathrm{Z}, \mathbf{H}_{1}\right), \mathbf{s} \in I^{3}$, соответственно.

I. Матрица кода $\mathbf{H}_{1}^{1}$ с параметром $r\left(\mathbf{H}_{1}^{1}\right)=0$ образуется путем допустимых преобразований представленной в таблице 1 матрицы 1) и имеет вид

$$
\mathbf{H}_{1}^{1}=\left(\begin{array}{rrrrrrrrrrr}
0 & 1 & 0 & 0 & -1 & 0 & 0 & 1 & -1 & -1 & 1 \\
0 & 0 & 1 & 0 & 0 & -1 & 0 & -1 & 1 & -1 & 1 \\
0 & 0 & 0 & 1 & 0 & 0 & -1 & -1 & -1 & 1 & 1
\end{array}\right)
$$

5 Дискретная математика, т. 26 № 4 
В соответствии с рассмотренным указанный $\mathrm{KB}$ является совершенным кодом, классифицирующим ситуации $Z=I_{2}^{11}$ на 27 равномощных подмножеств $W\left(\mathbf{s} \mid Z, \mathbf{H}_{1}^{1}\right)$, $\mathbf{s} \in I^{3}$, каждое из которых содержит по 9 элементов.

В множестве $I^{3}=S\left(Z, \mathbf{H}_{1}^{1}\right)$ всех $\left(\mathrm{Z}, \mathbf{H}_{1}^{1}\right)$-синдромов выделим подмножество $S_{b}=$ $\{(0,0,0),(1,0,0),(1,1,0),(1,1,1)\}$ так называемых базовых синдромов, на основе которых определим базовые множества $W\left(\mathbf{s}_{b} \mid Z, \mathbf{H}_{1}^{1}\right), \mathbf{s}_{b} \in S_{b}$.

Для базовых синдромов определим коды $\mathbf{H}_{2}^{1}\left(\mathbf{s}_{b}\right)=\left\langle\mathbf{h}^{4}, \mathbf{h}^{5}\right\rangle, \mathbf{s}_{b} \in S_{b},\left(r\left(\mathbf{H}_{2}^{1}\left(\mathbf{s}_{b}\right)=0\right)\right.$ проверочными матрицами, представленными в таблице 2.

Таблица 2

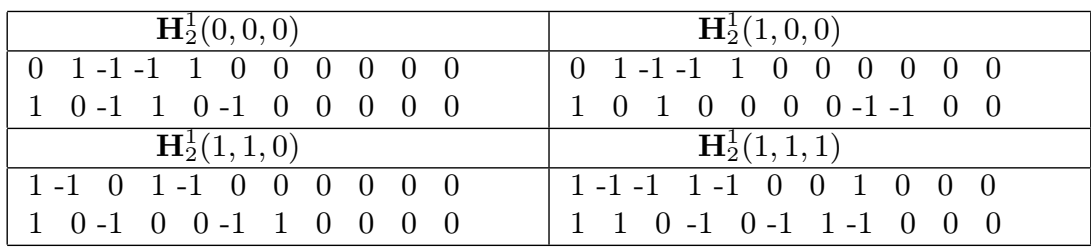

Последовательности, входящие в базовые множества $W\left(\mathbf{s}_{b} \mid Z, \mathbf{H}_{1}^{1}\right)$, а также синдромы этих последовательностей $\mathbf{s}\left(\mathbf{z} \mid \mathbf{H}_{2}^{1}\left(\mathbf{s}_{b}\right)\right)$ при $\mathbf{s}_{b} \in S_{b}$ для кодов $\mathbf{H}_{2}^{1}\left(\mathbf{s}_{b}\right)$, представлены в таблице 3 .

Таблица 3

\begin{tabular}{|c|c|c|c|c|}
\hline i & $W\left(0,0,0 \mid Z, \mathbf{H}_{1}^{\perp}\right)$ & $\mathbf{s}\left(\mathbf{z} \mid \mathbf{H}_{2}^{\perp}\right)$ & $W\left(1,0,0 \mid Z, \mathbf{H}_{1}^{\perp}\right)$ & $\mathbf{s}\left(\mathbf{z} \mid \mathbf{H}_{2}^{\perp}\right)$ \\
\hline 1 & $\begin{array}{llllllllllll}0 & 0 & 0 & 0 & 0 & 0 & 0 & 0 & 0 & 0 & 0\end{array}$ & $(0,0)$ & $\begin{array}{lllllllllll}0 & 0 & 0 & 0 & -1 & 0 & 0 & 0 & 0 & 0 & 0\end{array}$ & $(-1,0)$ \\
\hline 2 & 100000000000000 & $(0,1)$ & 010000000000000 & $(1,0)$ \\
\hline 3 & -10000000000000 & $(0,-1)$ & $\begin{array}{lllllllllll}0 & 0 & 0 & 0 & 0 & 0 & 0 & 1 & 0 & 0 & 1\end{array}$ & $(0,-1)$ \\
\hline 4 & 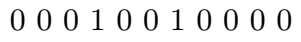 & $(-1,1)$ & $\begin{array}{lllllllll}0 & 0 & 0 & 0 & 0 & 0 & 0 & 0 & -1-1 \\
-1 & 0\end{array}$ & $(0,1)$ \\
\hline 5 & 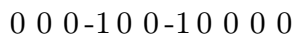 & $(1,-1)$ & $\begin{array}{lllllllllll}0 & 1 & 0 & 0 & -1 & 0 & 0 & 0 & 0 & 0 & 0\end{array}$ & $(0,0)$ \\
\hline 6 & $\begin{array}{lllllllllll}0 & 0 & 1 & 0 & 0 & 1 & 0 & 0 & 0 & 0 & 0\end{array}$ & $(-1,-1)$ & $10000-100000000$ & $(-1,0)$ \\
\hline 7 & 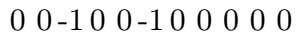 & $(1,1)$ & 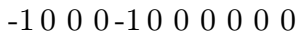 & $(-1,-1)$ \\
\hline 8 & 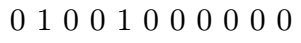 & $(1,0)$ & 1110000000000000 & $(1,1)$ \\
\hline 9 & $0-1000-100000000$ & $(-1,0)$ & -1110000000000000 & $(1,-1)$ \\
\hline i & $W\left(1,1,0 \mid Z, \mathbf{H}_{1}^{1}\right)$ & $\mathbf{s}\left(\mathbf{z} \mid \mathbf{H}_{2}^{1}\right)$ & $W\left(1,1,1 \mid Z, \mathbf{H}_{1}^{1}\right)$ & $\mathbf{s}\left(\mathbf{z} \mid \mathbf{H}_{2}^{1}\right)$ \\
\hline 1 & $\begin{array}{llllllllll}0 & 0 & 0 & 0 & 0 & 0 & 0 & 0 & 0 & -1\end{array}$ & $(0,0)$ & $\begin{array}{llllllllllll}0 & 0 & 0 & 0 & 0 & 0 & 0 & 0 & 0 & 0 & 1\end{array}$ & $(0,0)$ \\
\hline 2 & $\begin{array}{lllllllllll}0 & 0 & 0 & 0 & 0 & 0 & 1 & 0 & 0 & 0 & 1\end{array}$ & $(0,1)$ & $\begin{array}{lllllllllll}0 & 0 & 0 & 0 & 0 & 0 & -1 & 0 & 0 & 0 & 1\end{array}$ & $(0,-1)$ \\
\hline 3 & 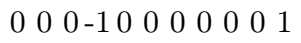 & $(-1,0)$ & $\begin{array}{lllllllllll}0 & 0 & 0 & 0 & 0 & -1 & 0 & 0 & 0 & 0 & 1\end{array}$ & $(0,1)$ \\
\hline 4 & $\begin{array}{lllllllllll}0 & 0 & 0 & 0 & 0 & 0 & -1 & 0 & 0 & -1 & 0\end{array}$ & $(0,-1)$ & 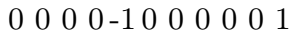 & $(1,0)$ \\
\hline 5 & 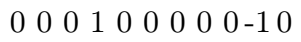 & $(1,0)$ & $\begin{array}{llllllllllll}0 & 0 & 0 & 1 & 0 & 0 & 0 & 0 & 0 & 0 & 1\end{array}$ & $(1,-1)$ \\
\hline 6 & $\begin{array}{llllllllllllll}0 & 0 & 0 & 0 & -1 & 0 & 0 & 0 & 0\end{array}$ & $(1,1)$ & $\begin{array}{lllllllllll}0 & 0 & 1 & 0 & 0 & 0 & 0 & 0 & 0 & 0 & 1\end{array}$ & $(-1,0)$ \\
\hline 7 & 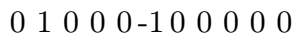 & $(-1,1)$ & $\begin{array}{llllllllllll}0 & 1 & 0 & 0 & 0 & 0 & 0 & 0 & 0 & 0 & 1\end{array}$ & $(-1,1)$ \\
\hline 8 & 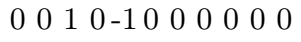 & $(1,-1)$ & 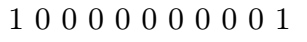 & $(1,1)$ \\
\hline 9 & 01110000000000 & $(-1,-1)$ & -1000000000001 & $(-1,-1)$ \\
\hline
\end{tabular}

Ниже показано, что множества $W\left(\mathbf{s} \mid Z, \mathbf{H}_{1}^{1}\right)$ и коды $\mathbf{H}_{2}^{1}(\mathbf{s})$ для всех синдромов $\mathbf{s} \in$ $I^{3}$ могут быть заданы на основе условных преобразований соответственно базовых множеств $W\left(\mathbf{s}_{b} \mid Z, \mathbf{H}_{1}^{1}\right), \mathbf{s}_{b} \in S_{b}$, и приведенных в таблице 2 кодов $\mathbf{H}_{2}^{1}\left(\mathbf{s}_{b}\right)$ для базовых синдромов $\mathbf{s}_{b} \in S_{b}$.

Для сокращения выкладок при задании перестановочных матриц $\mathbf{L} \in \mathcal{G}_{p}^{m}(\mathbf{R} \in$ $\left.\mathcal{G}_{p}^{m}\right)$ используем запись $\mathbf{L} \sim(p(1), p(2), \ldots, p(m))$, означающую, что в $i$-й строке матрицы $\mathbf{L}$ (соответственно $\mathbf{R}$ ) единица находится в столбце $p(i)$, а при задании диагональных матриц $\mathbf{L} \in \mathcal{G}_{d}^{m}\left(\mathbf{R} \in \mathcal{G}_{d}^{n}\right)$ - запись $\mathbf{L} \sim\left(c_{1}, c_{2}, \ldots c_{m}\right)$, означающую, что в матрице $\mathbf{L}$ (соответственно, $\mathbf{R}$ ) $i$-й диагональный элемент равен $c_{i}$. 
Отметим следующие важные для дальнейшего построения свойства кода $\mathbf{H}_{1}$. Пусть

$$
\begin{aligned}
& \mathbf{R}_{12} \sim(1,3,2,4,6,5,7,9,8,10,11), \mathbf{R}_{13} \sim(1,4,3,2,7,6,4,10,9,8,11), \\
& \mathbf{R}_{23} \sim(1,2,4,3,5,7,6,8,10,9,11), \mathbf{R}_{12}^{-} \sim(1,5,6,4,2,3,7,9,8,11,10), \\
& \mathbf{R}_{13}^{-} \sim(1,5,3,7,2,6,4,10,11,8,9), \mathbf{R}_{23}^{-} \sim(1,2,6,7,5,3,4,11,10,9,8)
\end{aligned}
$$

и $\mathbf{L}_{12} \sim(2,1,3), \mathbf{L}_{13} \sim(3,2,1), \mathbf{L}_{23} \sim(1,3,2), \mathbf{L}_{12}^{-} \sim(-1,-1,1), \mathbf{L}_{13}^{-} \sim(-1,1,-1)$, $\mathbf{L}_{23}^{-}(-1,-1,1)$.

Предложение 12. Для $\mathbf{L}_{i j} \in\left\{\mathbf{L}_{12}, \mathbf{L}_{13}, \mathbf{L}_{23}\right\}, \mathbf{R}_{i j} \in\left\{\mathbf{R}_{12}, \mathbf{R}_{13}, \mathbf{R}_{23}\right\}$ справедливо равенство $\mathbf{L}_{i j} \mathbf{H}_{1}^{1}=\mathbf{H}_{1}^{1} \mathbf{R}_{i j}$.

Предложение 13. Для $\mathbf{L}_{i j}^{-} \in\left\{\mathbf{L}_{12}^{-}, \mathbf{L}_{13}^{-}, \mathbf{L}_{23}^{-}\right\}, \mathbf{R}_{i j}^{-} \in\left\{\mathbf{R}_{12}^{-}, \mathbf{R}_{13}^{-}, \mathbf{R}_{23}^{-}\right\}$справедливо равенство $\mathbf{L}_{i j}^{-} \mathbf{H}_{1}^{1}=\mathbf{H}_{1}^{1} \mathbf{R}_{i j}^{-}$.

Пусть $\mathcal{L}_{b}=\left\{\mathbf{1},-\mathbf{1}, \mathbf{L}_{12}, \mathbf{L}_{13}, \mathbf{L}_{23}, \mathbf{L}_{12}^{-}, \mathbf{L}_{13}^{-}, \mathbf{L}_{23}^{-}\right\}, \quad \boldsymbol{R}_{b}=\left\{\mathbf{1},-\mathbf{1}, \mathbf{R}_{12}, \mathbf{R}_{13}\right.$, $\left.\mathbf{R}_{23}, \mathbf{R}_{12}^{-}, \mathbf{R}_{13}^{-}, \mathbf{R}_{23}^{-}\right\}, \mathcal{G}_{\mathbf{L}}^{m} \subseteq \mathcal{G}^{m}, \mathcal{G}_{\mathbf{R}}^{n} \subseteq \mathcal{G}^{n}-$ соответственно группы операторов, порожденных операторами из $\mathcal{L}_{b}$ и $\mathcal{R}_{b}$. При этом заметим, что предложения 12,13 определяют изоморфизм $\chi: \mathcal{G}_{\mathbf{L}}^{m} \rightarrow \mathcal{G}_{\mathbf{R}}^{n}\left(\chi^{-1}: \mathcal{G}_{\mathbf{R}}^{n} \rightarrow \mathcal{G}_{\mathbf{L}}^{m}\right)$.

Предложение 14. а) Все элементы $\mathbf{s} \in I^{3}$ представимы через базовые в виде $\mathbf{s}=$ $\mathbf{s}_{b} \mathbf{L}\left(\mathbf{s} \mid \mathbf{s}_{b}\right)$, где $\mathbf{L}\left(\mathbf{s} \mid \mathbf{s}_{b}\right) \in \mathcal{G}^{m}, \mathbf{s}_{b}$ определяется однозначно условием $w\left(\mathbf{s}_{b}\right)=w(\mathbf{s})$.

b) Все множества $W\left(\mathbf{s} \mid Z, \mathbf{H}_{1}^{1}\right), \mathbf{s} \in I^{3}$ представимы через базовые в виде $W\left(\mathbf{s} \mid Z, \mathbf{H}_{1}^{1}\right)=W\left(\mathbf{s}_{b} \mid Z, \mathbf{H}_{1}^{1}\right) \mathbf{R}\left(\mathbf{s} \mid \mathbf{s}_{b}\right)$, где $\mathbf{R}\left(\mathbf{s} \mid \mathbf{s}_{b}\right)=\chi \mathbf{L}\left(\mathbf{s} \mid \mathbf{s}_{b}\right), \mathbf{L}\left(\mathbf{s} \mid \mathbf{s}_{b}\right) \in \mathcal{G}^{m}$.

Варианты формул для операторов $\mathbf{L}\left(\mathbf{s} \mid \mathbf{s}_{b}\right)$ в виде композиции операторов из $\mathcal{L}_{b}$

\begin{tabular}{|c|c|c|c|c|c|c|c|c|}
\hline $\mathbf{S}$ & $\mathbf{s}_{b}$ & $\mathbf{L}\left(\mathbf{s} \mid \mathbf{s}_{b}\right)$ & $\mathrm{s}$ & $\mathbf{s}_{b}$ & $\mathbf{L}\left(\mathbf{s} \mid \mathbf{s}_{b}\right)$ & $\mathrm{s}$ & $\mathbf{s}_{b}$ & $\mathbf{L}\left(\mathbf{s} \mid \mathbf{s}_{b}\right)$ \\
\hline 000 & 000 & 1 & 100 & 100 & 1 & -100 & 100 & -1 \\
\hline 010 & 100 & $\mathbf{L}_{12}$ & 110 & 110 & 1 & -110 & 1110 & $\mathbf{L}_{13}^{-}$ \\
\hline $0-10$ & 100 & $-\mathbf{L}_{12}$ & $1-10$ & 110 & $\mathbf{L}_{23}^{-}$ & $-1-10$ & 110 & -1 \\
\hline 001 & 100 & $\mathbf{L}_{13}$ & 101 & 110 & $\mathbf{L}_{23}$ & $\begin{array}{lll}-1 & 0 & 1\end{array}$ & 110 & $\mathbf{L}_{23} \mathbf{L}_{12}^{-}$ \\
\hline 0111 & 110 & $\mathbf{L}_{13}$ & 111 & 111 & 1 & -111 & 111 & $-\mathbf{L}_{23}^{-}$ \\
\hline $0-11$ & 110 & $\mathbf{L}_{13} \mathbf{L}_{12}^{-}$ & $1-11$ & 111 & $-\mathbf{L}_{13}^{-}$ & $-1-11$ & 111 & $\mathbf{L}_{12}^{-}$ \\
\hline $\begin{array}{lll}0 & 0 & -1\end{array}$ & 100 & $-\mathbf{L}_{13}$ & $10-1$ & 110 & $\mathbf{L}_{23} \mathbf{L}_{23}^{-}$ & $\begin{array}{lll}-1 & 0 & -1\end{array}$ & 110 & $-\mathbf{L}_{23}$ \\
\hline $01-1$ & 110 & $\mathbf{L}_{13} \mathbf{L}_{13}^{-}$ & $11-1$ & 111 & $-\mathbf{L}_{12}^{-}$ & $-11-1$ & 111 & $\mathbf{L}_{13}^{-}$ \\
\hline $0-1-1$ & 110 & $-\mathbf{L}_{13}$ & $1-1-1$ & 111 & $\mathbf{L}_{23}^{-}$ & $-1-1-1$ & 111 & -1 \\
\hline
\end{tabular}
(доказывающие предложение 14) представлены в таблице 4.

\section{Таблица 4}

Таким образом, все 27 множеств $W\left(\mathbf{s} \mid Z, \mathbf{H}_{1}^{1}\right), \mathbf{s} \in I^{3}$, могут быть получены путем преобразований $\mathbf{R}\left(\mathbf{s} \mid \mathbf{s}_{b}\right) \in \mathcal{R}_{b}$ четырех множеств $W\left(\mathbf{s}_{b} \mid Z, \mathbf{H}_{1}^{1}\right)$ для $\mathbf{s}_{b} \in S_{b}$ в виде $W\left(\mathbf{s} \mid Z, \mathbf{H}_{1}^{1}\right)=W\left(\mathbf{s}_{b} \mid Z, \mathbf{H}_{1}^{1}\right) \mathbf{R}\left(\mathbf{s} \mid \mathbf{s}_{b}\right)$, где $\mathbf{R}\left(\mathbf{s} \mid \mathbf{s}_{b}\right)=\chi \mathbf{L}\left(\mathbf{s} \mid \mathbf{s}_{b}\right)$

Предложение 15. Пусть $\mathbf{s}^{1}=\mathbf{s}_{b}^{1} \mathbf{L}\left(\mathbf{s}^{1} \mid \mathbf{s}_{b}^{1}\right), \mathbf{s}^{2}=\mathbf{s}_{b}^{2} \mathbf{L}\left(\mathbf{s}^{2} \mid \mathbf{s}_{b}^{2}\right)$. Тогда $\mathbf{s}^{1}=\mathbf{s}^{2} \Leftrightarrow \mathbf{s}_{b}^{1}=\mathbf{s}_{b}^{2}$.

Доказательство. 1) Прямое утверждение. Заметим, что $w\left(\mathbf{s}^{1}\right)=w\left(\mathbf{s}_{b}^{1} \mathbf{L}\left(\mathbf{s}^{1} \mid \mathbf{s}_{b}^{1}\right)=\right.$ $w\left(\mathbf{s}_{b}^{1}\right)$. Аналогично $w\left(\mathbf{s}^{2}\right)=w\left(\mathbf{s}_{b}^{2}\right)$. Пусть $\mathbf{s}_{b}^{1} \neq \mathbf{s}_{b}^{2}$. Тогда, учитывая, что все элементы $S_{b}$ различаются по весу Хемминга, получим $w\left(\mathbf{s}_{b}^{1}\right) \neq w\left(\mathbf{s}_{b}^{2}\right) \Rightarrow w\left(\mathbf{s}^{1}\right) \neq w\left(\mathbf{s}^{2}\right) \Rightarrow \mathbf{s}^{1} \neq$ $\mathbf{s}^{2}$. Таким образом, из $\mathbf{s}^{1}=\mathbf{s}^{2}$ вытекает $\mathbf{s}_{b}^{1}=\mathbf{s}_{b}^{2}$. 2) Обратное утверждение очевидно.

Теорема 4. $A B \mathcal{A}^{1}=\left\langle\mathbf{H}_{1}^{1}, \mathbf{H}_{2}^{1}(\mathbf{s})\right\rangle$, где $\mathbf{s}=\mathbf{s}\left(\mathbf{z} \mid \mathbf{H}_{1}^{1}\right), \mathbf{z} \in I_{2}^{11},-$ совершенный $(11,5,2)$-алгоритм. 
II. Матрица кода $\mathbf{H}_{1}^{2}$ с параметром $r\left(\mathbf{H}_{1}^{2}\right)=0$ образуется путем допустимых преобразований представленной в таблице 1 матрицы 2) и имеет вид

$$
\mathbf{H}_{1}^{2}=\left(\begin{array}{rrrrrrrrrrr}
0 & 1 & -1 & 0 & -1 & 0 & 0 & 1 & 1 & -1 & -1 \\
0 & 0 & 0 & 1 & 1 & -1 & -1 & 0 & 0 & 1 & -1 \\
0 & 0 & 0 & 1 & -1 & 1 & -1 & 1 & -1 & 0 & 0
\end{array}\right)
$$

Далее, аналогично рассмотренному в п. I, введем преобразования $-\mathbf{R}_{23} \sim(1,3,2,7,5,6,4,11,10,9,8), \quad \mathbf{R}_{2}^{-} \sim(1,2,3,6,7,4,5,8,9,11,10), \quad \mathbf{R}_{3}^{-} \sim$ $(1,2,3,5,4,7,6,9,8,10,11)$ и $\mathbf{L}_{23} \sim(1,3,2), \mathbf{L}_{2}^{-} \sim(1,-1,1), \mathbf{L}_{3}^{-} \sim(1,1,-1)$.

Предложение 16. а) $\mathbf{L}_{23} \mathbf{H}_{1}^{2}=\mathbf{H}_{1}^{2} \mathbf{R}_{23} ;$ b) $\mathbf{L}_{2}^{-} \mathbf{H}_{1}^{2}=\mathbf{H}_{1}^{2} \mathbf{R}_{2}^{-}$; c) $\mathbf{L}_{3}^{-} \mathbf{H}_{1}^{2}=\mathbf{H}_{1}^{2} \mathbf{R}_{3}^{-}$.

Определим множество базовых синдромов $S_{b}=\{(0,0,0),(1,0,0),(0,1,0),(1,1,0)$, $(0,1,1),(1,1,1)\}$ и базовые множества $W\left(\mathbf{s}_{b} \mid Z, \mathbf{H}_{1}^{2}\right), \mathbf{s}_{b} \in S_{b}$. Обозначим $\mathcal{L}_{b}=$ $\left\{\mathbf{1},-\mathbf{1}, \mathbf{L}_{23}, \mathbf{L}_{2}^{-}, \mathbf{L}_{3}^{-}\right\}, \mathcal{R}_{b}=\left\{\mathbf{1},-\mathbf{1}, \mathbf{R}_{23}, \mathbf{R}_{2}^{-}, \mathbf{R}_{3}^{-}\right\}$, пусть $\mathcal{G}_{\mathbf{L}}^{m} \subseteq \mathcal{G}^{m}, \mathcal{G}_{\mathbf{R}}^{n} \subseteq \mathcal{G}^{n}-$ соответственно группы операторов, порожденных операторами из $\mathcal{L}_{b}$ и $\boldsymbol{\mathcal { R }}_{b}$, а изоморфизм $\chi: \mathcal{G}_{\mathbf{L}}^{m} \rightarrow \mathcal{G}_{\mathbf{R}}^{n}\left(\chi^{-1}: \mathcal{G}_{\mathbf{R}}^{n} \rightarrow \mathcal{G}_{\mathbf{L}}^{m}\right)$ определен равенствами предложения 16 .

Коды $\mathbf{H}_{2}^{2}\left(\mathbf{s}_{b}\right)=\left\langle\mathbf{h}^{4}, \mathbf{h}^{5}\right\rangle$ (с параметром $r\left(\mathbf{H}_{2}^{2}\left(\mathbf{s}_{b}\right)\right)=0$ ) для базовых синдромов $\mathbf{s}_{b} \in S_{b}$ представлены проверочными матрицами в таблице 5 .

\section{Таблица 5}

\begin{tabular}{|c|c|c|}
\hline $\mathbf{H}_{2}^{2}(0,0,0)$ & $\mathbf{H}_{2}^{2}(1,0,0)$ & $\mathbf{H}_{2}^{2}(0,1,0)$ \\
\hline $1-1-11000000000$ & $\begin{array}{llllllllllll}0 & 1 & 1 & -1 & -1 & 0 & 0 & 0 & 0 & 0 & 0\end{array}$ & $\begin{array}{lllllllllll}0 & 1 & -1 & -1 & 1 & 0 & 0 & 0 & 0 & 0 & 0\end{array}$ \\
\hline 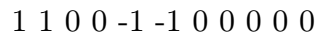 & $\begin{array}{llllllllllllllllllll}1 & 0 & 0 & 1 & 0 & 0 & 0 & -1 & 0 & -1 & 0\end{array}$ & 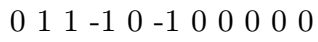 \\
\hline $\mathbf{H}_{2}^{2}(1,1,0)$ & $\mathbf{H}_{2}^{2}(0,1,1)$ & $\mathbf{H}_{2}^{2}(1,1,1)$ \\
\hline $\begin{array}{lllllllllll}1 & 0 & -1 & 1 & 0 & 0 & 0 & -1 & 0 & 0 & 0\end{array}$ & $\begin{array}{lllllllllll}0 & 0 & 0 & 1 & -1 & -1 & 1 & 0 & 0 & 0 & 0\end{array}$ & $\begin{array}{llllllllllll}0 & 1 & 1 & 0 & 0 & 0 & -1 & -1 & 0 & 0 & 0\end{array}$ \\
\hline 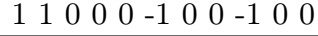 & 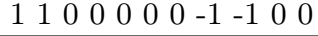 & $\begin{array}{lllllllllll}0 & 0 & 1 & -1 & 0 & 0 & 0 & 1 & 0 & 0 & -1\end{array}$ \\
\hline
\end{tabular}

Последовательности, входящие в базовые множества $W\left(\mathbf{s}_{b} \mid Z, \mathbf{H}_{1}^{2}\right)$, а также синдромы этих последовательностей $\mathbf{s}\left(\mathbf{z} \mid \mathbf{H}_{2}^{2}\left(\mathbf{s}_{b}\right)\right)$ при $\mathbf{s}_{b} \in S_{b}$ для кодов $\mathbf{H}_{2}^{2}\left(\mathbf{s}_{b}\right)$ представлены в таблице 6 .

\section{Таблица 6}

\begin{tabular}{|c|c|c|c|c|}
\hline i & $\overline{W\left(0,0,0 \mid Z, \mathbf{H}_{1}^{2}\right)}$ & $\mathbf{s}\left(\mathbf{z} \mid \mathbf{H}_{2}^{2}\right)$ & $W\left(1,0,0 \mid Z, \mathbf{H}_{1}^{2}\right)$ & $\mathbf{s}\left(\mathbf{z} \mid \mathbf{H}_{2}^{2}\right)$ \\
\hline 1 & $\begin{array}{llllllllllll}0 & 0 & 0 & 0 & 0 & 0 & 0 & 0 & 0 & 0 & 0\end{array}$ & $(0,0)$ & $\begin{array}{llllllllll}0 & 0 & -1 & 0 & 0 & 0 & 0 & 0 & 0 & 0\end{array}$ & $(-1,0)$ \\
\hline 2 & $\begin{array}{llllllllllll}1 & 0 & 0 & 0 & 0 & 0 & 0 & 0 & 0 & 0 & 0\end{array}$ & $(1,1)$ & $\begin{array}{llllllllllll}0 & 1 & 0 & 0 & 0 & 0 & 0 & 0 & 0 & 0 & 0\end{array}$ & $(1,0)$ \\
\hline 3 & -100000000000 & $(-1,-1)$ & $\begin{array}{llllllllll}0 & 0 & 0 & 0 & 0 & 0 & 0 & 0 & 0 & -1-1\end{array}$ & $(0,1)$ \\
\hline 4 & 000010001000000 & $(1,0)$ & 00000000001100 & $(0,-1)$ \\
\hline 5 & 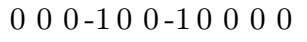 & $(-1,0)$ & 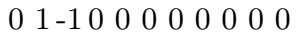 & $(0,0)$ \\
\hline 6 & 0000001110000000 & $(0,-1)$ & $10 \begin{array}{lllllllll}1 & 0 & 0 & 0 & 0 & 0 & 0 & 0 & 0\end{array}$ & $(-1,1)$ \\
\hline 7 & 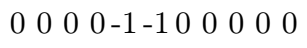 & $(0,1)$ & $-10-100000000$ & $(-1,-1)$ \\
\hline 8 & 0111000000000000 & $(-1,1)$ & 1110000000000000 & $(1,1)$ \\
\hline 9 & $0-1-1000000000$ & $(1,-1)$ & 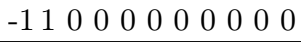 & $(1,-1)$ \\
\hline
\end{tabular}




\begin{tabular}{|c|c|c|c|c|}
\hline $\mathrm{i}$ & $W\left(0,1,0 \mid Z, \mathbf{H}_{1}^{2}\right)$ & $\mathbf{s}\left(\mathbf{z} \mid \mathbf{H}_{2}^{2}\right)$ & $W\left(1,1,0 \mid Z, \mathbf{H}_{1}^{2}\right.$ & $\mathbf{s}\left(\mathbf{z} \mid \mathbf{H}_{2}^{2}\right)$ \\
\hline 1 & $\begin{array}{lllllllllll}0 & 0 & 0 & 0 & 0 & 0 & 0 & 0 & 0 & 1 & -1\end{array}$ & $(0,0)$ & $\begin{array}{llllllllllllll}0 & 0 & 0 & 0 & 0 & 0 & 0 & 0 & 0 & 0 & -1\end{array}$ & $(0,0)$ \\
\hline 2 & $\begin{array}{lllllllllll}0 & 0 & 1 & 0 & 0 & 0 & 0 & 0 & 0 & 0 & -1\end{array}$ & $(-1,1)$ & 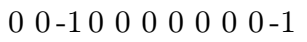 & $(1,0)$ \\
\hline 3 & $0-100000000000-1$ & $(-1,-1)$ & $\begin{array}{lllllllllll}0 & 1 & 0 & 0 & 0 & 0 & 0 & 0 & 0 & 0 & -1\end{array}$ & $(0,1)$ \\
\hline 4 & 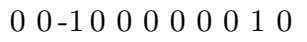 & $(1,-1)$ & 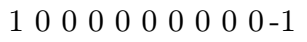 & $(1,1)$ \\
\hline 5 & 010000000000010 & $(1,1)$ & $-100000000000-1$ & $(-1,-1)$ \\
\hline 6 & $\begin{array}{lllllllllll}0 & 0 & 0 & 0 & 0 & -1 & -1 & 0 & 0 & 0 & 0\end{array}$ & $(0,1)$ & $\begin{array}{lllllllllll}0 & 0 & 0 & 0 & 0 & 0 & -1 & 0 & 1 & 0 & 0\end{array}$ & $(0,-1)$ \\
\hline 7 & 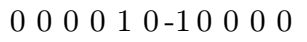 & $(1,0)$ & 000010000001000 & $(1,-1)$ \\
\hline 8 & 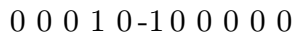 & $(-1,0)$ & $\begin{array}{lllllllllll}0 & 0 & 0 & 0 & 0 & -1 & 0 & 1 & 0 & 0 & 0\end{array}$ & $(-1,1)$ \\
\hline 9 & 000011100000000 & $(0,-1)$ & 00000100010000 & $(-1,0)$ \\
\hline i & $\left.W(0,1,1) \mid Z, \mathbf{H}_{1}^{2}\right)$ & $\mathbf{s}\left(\mathbf{z} \mid \mathbf{H}_{2}^{2}\right)$ & $W\left(1,1,1 \mid Z, \mathbf{H}_{1}^{2}\right)$ & $\mathbf{s}\left(\mathbf{z} \mid \mathbf{H}_{2}^{2}\right)$ \\
\hline 1 & $\begin{array}{lllllllllll}0 & 0 & 0 & 0 & 0 & 0 & -1 & 0 & 0 & 0 & 0\end{array}$ & $(-1,0)$ & $\begin{array}{lllllllllll}0 & 0 & 0 & 0 & 0 & 0 & 0 & 1 & 0 & 0 & -1\end{array}$ & $(-1,1)$ \\
\hline 2 & 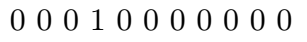 & $(1,0)$ & 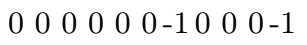 & $(1,1)$ \\
\hline 3 & $\begin{array}{lllllllllll}0 & 0 & 0 & 0 & 0 & 0 & 0 & 0 & -1 & 0 & -1\end{array}$ & $(0,1)$ & $\begin{array}{lllllllllll}0 & 0 & 0 & 1 & 0 & 0 & 0 & 0 & 0 & 0 & -1\end{array}$ & $(0,0)$ \\
\hline 4 & $\begin{array}{lllllllllll}0 & 0 & 0 & 0 & 0 & 0 & 0 & 1 & 0 & 1 & 0\end{array}$ & $(0,-1)$ & $\begin{array}{lllllllllll}0 & 0 & 0 & 0 & 0 & 0 & -1 & 1 & 0 & 0 & 0\end{array}$ & $(0,1)$ \\
\hline 5 & 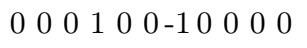 & $(0,0)$ & $\begin{array}{lllllllllll}0 & 0 & 0 & 1 & 0 & 0 & 0 & 1 & 0 & 0 & 0\end{array}$ & $(-1,0)$ \\
\hline 6 & $10000000-1100000$ & $(-1,1)$ & 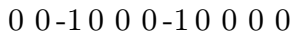 & $(0,-1)$ \\
\hline 7 & $-1000000-10000$ & $(-1,-1)$ & $\begin{array}{lllllllllll}0 & 1 & 0 & 0 & 0 & 0 & -1 & 0 & 0 & 0 & 0\end{array}$ & $(1,0)$ \\
\hline 8 & $\begin{array}{llllllllllll}1 & 0 & 0 & 1 & 0 & 0 & 0 & 0 & 0 & 0 & 0\end{array}$ & $(1,1)$ & $\begin{array}{llllllllllll}0 & 0 & -1 & 1 & 0 & 0 & 0 & 0 & 0 & 0 & 0\end{array}$ & $(-1,-1)$ \\
\hline 9 & -10001000000000 & $(1,-1)$ & 010010000000000 & $(1,-1)$ \\
\hline
\end{tabular}

Варианты формул для операторов $\mathbf{L}\left(\mathbf{s} \mid \mathbf{s}_{b}\right)$ в виде композиции операторов из $\mathcal{L}_{b}$ представлены в таблице 7.

\section{Таблица 7}

\begin{tabular}{|c|c|c|c|c|c|c|c|c|}
\hline $\mathbf{s}$ & $\mathbf{s}_{b}$ & $\mathbf{L}\left(\mathbf{s} \mid \mathbf{s}_{b}\right)$ & $\mathbf{s}$ & $\mathbf{s}_{b}$ & $\mathbf{L}\left(\mathbf{s} \mid \mathbf{s}_{b}\right)$ & $\mathbf{s}$ & $\mathbf{s}_{b}$ & $\mathbf{L}\left(\mathbf{s} \mid \mathbf{s}_{b}\right)$ \\
\hline $\begin{array}{llll}0 & 0 & 0\end{array}$ & 0000 & 1 & 100 & 100 & 1 & -100 & 100 & -1 \\
\hline 010 & 010 & 1 & 110 & 110 & 1 & -110 & 110 & $-\mathbf{L}_{2}^{-}$ \\
\hline $0-10$ & 010 & -1 & $1-10$ & 110 & $\mathbf{L}_{2}^{-}$ & $-1-10$ & 110 & -1 \\
\hline $\begin{array}{llll}0 & 0 & 1\end{array}$ & 010 & $\mathbf{L}_{23}$ & 101 & 110 & $\mathbf{L}_{23}$ & $\begin{array}{llll}-1 & 0 & 1\end{array}$ & 110 & $-\mathbf{L}_{3} \mathbf{L}_{23}$ \\
\hline 0111 & 011 & 1 & 111 & 111 & 1 & -111 & 111 & $-\mathbf{L}_{3}^{-} \mathbf{L}_{2}^{-}$ \\
\hline $0-11$ & 011 & $\overline{\mathbf{L}_{2}^{-}}$ & $1-11$ & 111 & $\mathbf{L}_{2}^{-}$ & $-1-11$ & 111 & $-\mathbf{L}_{3}^{-}$ \\
\hline $\begin{array}{lll}0 & 0 & -1 \\
\end{array}$ & 010 & $-\mathbf{L}_{23}$ & $10-1$ & 110 & $\mathbf{L}_{3}^{-} \mathbf{L}_{23}$ & $-10-1$ & 110 & $-\mathbf{L}_{23}$ \\
\hline 0 1-1 & 011 & $\mathbf{L}_{3}^{-}$ & $11-1$ & 111 & $\mathbf{L}_{3}^{-}$ & $-11-1$ & 111 & $-\mathbf{L}_{2}^{-}$ \\
\hline $0-1-1$ & 011 & -1 & $1-1-1$ & 111 & $\mathbf{L}_{2}^{-} \mathbf{L}_{3}^{-}$ & $-1-1-1$ & 111 & -1 \\
\hline
\end{tabular}

Теорема 5. $A B \mathcal{A}^{2}=\left\langle\mathbf{H}_{1}^{2}, \mathbf{H}_{2}^{2}(\mathbf{s})\right\rangle$, где $\mathbf{s}=\mathbf{s}\left(\mathbf{z} \mid \mathbf{H}_{1}^{2}\right), \mathbf{z} \in I_{2}^{11}, \quad-$ совершенный $(11,5,2)$-алгоритм.

\section{6. Заключение}

В заключение обсудим проблему получения верхней границы для минимального числа взвешиваний, при котором гарантируется существование алгоритмов классификации и идентификации. В силу того, что в результате каждого взвешивания меняется множество допустимых ситуаций, классификацию которых необходимо осуществлять следующим взвешиванием, интерес представляет получение так называемой универсальной границы, под которой понимается неравенство, связывающее параметры взвешивания, при выполнении которого гарантируется существование взвешивания с такими параметрами в любом множестве ситуаций $Z \subseteq I^{n}$. 
Гарантированный диаметр классификации взвешиванием определяется выражением

$$
\begin{aligned}
& d(M, n)=\max _{Z \subseteq I^{n}} \min _{\mathbf{h} \in I^{n}} d(Z, \mathbf{h})= \\
& =\max _{Z \subseteq I^{n}} \min _{\mathbf{h} \in I^{n}} \max \{M(0 \mid Z, \mathbf{h}), M(1 \mid Z, \mathbf{h}), M(-1 \mid Z, \mathbf{h})\}, \quad \text { где } M=|Z| .
\end{aligned}
$$

С геометрической точки зрения величина $d(M, n)$ определяет гарантированный (достижимый) диаметр разбиения любого множества $Z=\left\{\mathbf{z}^{i}\right\} \subseteq I^{n}(|Z|=M)$ плоскостью $[\mathbf{z}, \mathbf{h}]=0$ при $\mathbf{h} \in I^{n}$ на три части $\left\{\mathbf{z} \in I^{n} \mid[\mathbf{z}, \mathbf{h}]<0\right\},\left\{\mathbf{z} \in I^{n} \mid[\mathbf{z}, \mathbf{h}]>0\right\}$ и $\left\{\mathbf{z} \in I^{n} \mid[\mathbf{z}, \mathbf{h}]=0\right\}$; при этом гарантируется, что мощность максимальной части не превысит $d(M, n)$. Аналогичным образом определяется и интерпретируется величина $d_{0}(M, n)$ для множества центрированных взвешиваний.

Для $M \leqslant 10$ установлены следующие значения величин $d(M, n): d(2, n)=1$; $d(3,1)=1, d(3, n)=2$ при $n \geqslant 2 ; d(4, n)=2$ при $n \geqslant 2 ; d(5,2)=2, d(5, n)=3$ при $n \geqslant 3 ; d(6, n)=3$ при $n \geqslant 2 ; d(7, n)=3$ при $n \geqslant 2 ; d(8,2)=3, d(8, n)=4$ при $n \geqslant 3$; $d(9,2)=3, d(9, n)=4$ при $n \geqslant 3 ; d(10, n)=5$ при $n \geqslant 3$, а также величин $d_{0}(M, n)$ : $d_{0}(M, n)=2$ при $n \geqslant 2 ; d_{0}(3, n)=3$ при $n \geqslant 2 ; d_{0}(4, n)=3$ при $n \geqslant 2 ; d_{0}(5,2)=3$, $d_{0}(5, n)=4$ при $n \geqslant 3 ; d_{0}(6,2)=3, d_{0}(6, n)=4$ при $n \geqslant 3 ; d_{0}(7,2)=3, d_{0}(7, n)=5$ при $n \geqslant 3 ; d_{0}(8,2)=3, d_{0}(8, n)=5$ при $n \geqslant 3 ; d_{0}(9,2)=3, d_{0}(9, n)=6$ при $n \geqslant 3$; $d_{0}(10, n)=6$ при $n \geqslant 3$.

Есть основания полагать, что $\lim \sup _{M \rightarrow \infty} \frac{d_{0}(M, n)}{M}=\lim \sup _{M \rightarrow \infty} \frac{d(M, n)}{M}=\frac{1}{3}$, причем при всех $M \geqslant 6$ справедливо неравенство $\frac{d(M, n)}{M} \leqslant \frac{1}{2}$, однако на настоящий момент этот вопрос остается открытым. Наличие эффективной границы вида $d(M, n) / M k$ позволило бы получать оценки числа взвешиваний, достаточного для классификации и идентификации ситуаций в любом подмножестве $I^{n}$.

\section{Список литературы}

1. Яглом А. М., Яглом И. М., Вероятность и информачия, Наука, Москва, 1973.

2. Канель-Белов А. Я., Френкин Б. Р., “Дополнение к статье Д. А .Михалина, И. М. Никонова «Одна задача о нахождении фальшивой монеты»", Математическое просвещение, 3:12, 229-231.

3. Alon N., Kozlov D. N., Vu V. H., "The geometry of coin-weighing problems", Proc. 37th Annu. Found. Comput. Sci. (Burlington), 524-532.

4. Bshouty N. H., "Optimal algorithms for the coin weighing problem with a spring scale", COLT 2009 Proceedings, 34-43.

5. Berlekamp E. R., PhD. Thesis, Massachusetts Inst. of Technology, Dept. of Electr. Eng., 1964.

6. Бассалыго Л. А., "Недвоичные коды, исправляющие ошибки при наличии одноразовой безошибочной обратной связи", Проблемы передачи информации, 41:2 (2009), 63-67.

7. Barg A., "At the dawn of the theory of codes", The Math. Intelligencer, 15:1 (1993), 20-26.

8. Murota K., Discrete Convex Analysis, SIAM, Philadelphia, 2003.

9. Данилов В.И, Кошевой Г.А., "Дискретная выпуклость", Записки научных семинаров ПОМИ, 312 (2004), 86-93. 